\title{
FORCA, a promoter element that responds to crosstalk between defense and light signaling
}

\author{
Alexandre Evrard ${ }^{1,2}$, Theogene Ndatimana ${ }^{1}$ and Thomas Eulgem*1
} Address: ${ }^{1}$ Center for Plant Cell Biology, Institute for Integrative Genome Biology, Department of Botany and Plant Sciences, University of
California, Riverside, CA 92521, USA and ${ }^{2}$ INRA/CNRS-URGV, 2 Rue Gaston Crémieux, CP5708 91057, Evry, France

Email: Alexandre Evrard - alevrard@evry.inra.fr; Theogene Ndatimana - tndat001@student.ucr.edu; Thomas Eulgem* - thomas.eulgem@ucr.edu

* Corresponding author

Published: 7 January 2009

BMC Plant Biology 2009, 9:2 doi:10.1 I86/147/-2229-9-2

This article is available from: http://www.biomedcentral.com/I47I-2229/9/2

(C) 2009 Evrard et al; licensee BioMed Central Ltd.

This is an Open Access article distributed under the terms of the Creative Commons Attribution License (http://creativecommons.org/licenses/by/2.0), which permits unrestricted use, distribution, and reproduction in any medium, provided the original work is properly cited.
Received: 10 September 2008

Accepted: 7 January 2009

\begin{abstract}
Background: Recognition of pathogenic microorganisms triggers in plants comprehensive transcriptional reprogramming. In order to identify transcriptome-level control elements required for plant immune responses we are examining several sets of genes found by microarray experiments to be co-activated in Arabidopsis thaliana (Arabidopsis) seedlings infected with the oomycete Hyaloperonospora parasitica. Promoter motifs conserved in clusters of co-expressed genes may be involved in mediating coordinated gene activity patterns. Although numerous studies identified such conserved promoter motifs in co-expressed gene sets, reports confirming their function as regulatory elements are rare.
\end{abstract}

Results: FORCA is a hexameric promoter motif that is conserved in clusters of Arabidopsis genes co-expressed in response to fungal or oomycete pathogens as well as defined light treatments. FORCA is generally more frequently present in Arabidopsis promoter regions than statistically expected. It constitutively interacts in a DNA-sequence specific manner with nuclear Arabidopsis proteins. These interactions are suppressed by defense-related stimuli and enhanced by prolonged exposure to constant light. Furthermore FORCA mediates constitutive reporter gene expression in transiently transformed Nicotiana benthamiana leaves as well as in stably transformed Arabidopsis plants. Its responsiveness to defense-stimuli is modulated by the duration of light exposure. In plants grown under normal light conditions or constant darkness defense-related stimuli result in suppression of FORCA-mediated reporter gene expression, while in plants grown under constant light exposure, defense-induction results in enhanced FORCA-mediated expression. In addition, we found plants subjected to constant light exposure to exhibit reduced susceptibility to virulent $H$. parasitica.

Conclusion: We propose that FORCA is a regulatory cis-element that is present in a wide variety of Arabidopsis promoters. It integrates light- and defense-related signals and participates in adjusting the transcriptome to changes in environmental conditions.

\section{Background}

Molecular recognition of pathogenic microorganisms triggers in plants comprehensive transcriptional reprogramming. A network of defense-regulators transduces information about the attacking microbe into appropriate transcriptional responses. Two functionally distinct classes of pathogen molecules are known to elicit plant immune responses [1-3]. Receptor-mediated recognition 
of pathogen/microbe associated molecular patterns (PAMPs/MAMPs) activates PAMP-triggered immunity (PTI), while recognition of pathogen effectors by plant disease resistance $(\mathrm{R})$ proteins leads to effector-triggered immunity (ETI). ETI is a strong immune response that results in incompatible plant pathogen interactions where the plant is resistant and the pathogen is avirulent. Effectors are pathogen-derived molecules (proteins or small organic molecules) that are secreted into host tissues [4]. Some effectors have been shown to suppress PTI resulting in a weakened immune response called basal defense which is usually insufficient to halt growth and spread of the respective microbe. The typical outcome in this case is a compatible interaction, where the plant is susceptible and the pathogen is virulent.

A key player in the regulation of ETI as well as basal defense is the hormone salicylic acid (SA), which accumulates in infected tissues of several plant species and triggers downstream defense responses [5]. Several other small molecules and multiple proteins involved in defense regulation have been identified by genetic and biochemical studies [6]. In particular the immune system of Arabidopsis thaliana (Arabidopsis) has been extensively examined over the past two to three decades. While our knowledge about the Arabidopsis immune system is far from complete, it has become obvious in recent years, that its individual components are functionally interconnected in a complex manner and constitute a regulatory network rather than simple linear pathways. Crosstalk between distinct branches of this network has been found to be involved in fine-tuning defense outputs and maximizing the effectiveness of immune responses [7]. For example, sophisticated mechanisms mediating crosstalk between SA and jasmonic acid (JA)-dependent signaling processes can result in synergistic or antagonistic effects depending on the levels of each of these hormones [8-10].

In addition, there are extensive interactions of defense signaling with regulatory processes that are not primarily involved in plant immune responses. For example, crosstalk between defense mechanisms dependent on the defense hormones SA and JA on the one hand and each of the classical growth-controlling phytohormones, auxin, gibberellin, ABA, cytokinin or brassinosteroids on the other hand have been reported [11]. Details of these interactions are not well understood yet, but it seems that some of them benefit the host by enhancing immune responses, while others are beneficial for the pathogen by suppressing defense reactions. In addition, several labs reported interferences between light and defense signaling. Depending on the type of light signaling pathway involved, these interactions were found to be synergistic or antagonistic. For example, phytochrome-mediated light signaling can positively affect SA-dependent defense responses [12,13], while negative interferences were observed between defense and UV-light signaling [14]. Generally, these interactions seem to control resource allocation in the plant ensuring the maintenance of homeostasis and the adjustment of the host's metabolism to a state optimal to cope with the respective environmental challenges.

We are using interactions between the pathogenic oomycete Hyaloperonospora parasitica $(H p)$ and Arabidopsis to examine regulatory mechanisms operating at the interface between defense signaling and the regulation of the defense transcriptome [15-17]. Several transcriptional regulators have been implicated in these processes, such as NPR1, a nuclear transported transcriptional cofactor that acts downstream from SA and interacts with TGA-bZIP transcription factors [18]. Additional transcription factors, including WRKYs and ERFs, participate in the regulation of the plant defense transcriptome and disease resistance [19]. The DNA binding site preferences of these transcription factors have been well characterized. Typically TGA-bZIPs bind to TGA boxes (TGACG), WRKYs to $\mathrm{W}$ boxes (TTGACC/T) and ERFs to GCC boxes (AGCCGCC) [19]. These promoter sites have been shown in numerous studies to act as pathogen-responsive cis-elements mediating expression of individual defense-associated genes [19].

An enormous amount of global gene expression data from large-scale transcript profiling projects has accumulated during the past decade. These data allowed for the identification of gene clusters that are co-expressed under certain biological conditions and, hence, are likely to be subject to co-regulation by common mechanisms. Several studies revealed conserved sequence motifs in promoters of such co-regulated genes which may act as cis-elements mediating their coordinated activity $[20,21]$. In addition to already known cis-elements, some novel promoter motifs were found to be statistically enriched in coexpressed genes $[15,22,23]$. While in some cases, their function has been experimentally proven $[22,23]$, reports confirming that such conserved promoter motifs are binding sites of nuclear proteins that can affect gene expression are still rare.

Here we report on the identification and functional characterization of a motif conserved in promoters of the FORC cluster, a set of Arabidopsis genes co-expressed in response to recognition of $\mathrm{Hp}$ as well as other oomycete and fungal pathogens. We found this motif, which we termed FORCA, to exhibit DNA sequence specific and differential interactions with nuclear Arabidopsis proteins. Furthermore, electrophoretic mobility shift assays as well as reporter gene assays showed that FORC $C^{\mathrm{A}}$ integrates stimuli related to defense- and light signaling. FORC $C^{\mathrm{A}}$ acts 
as a light-responsive enhancer, the activity of which is modulated by pathogen-perception. Under normal light conditions as well as constant darkness, FORC A activity is reduced by recognition of $H p$ as well as application of SA, while under constant light conditions its activity is enhanced by defense induction. Consistent with the observed functional connection between light and defense signaling, we observed that plants kept under constant light exposure exhibited reduced susceptibility to a virulent $H p$ isolate.

\section{Results}

\section{A cluster of Arabidopsis genes co-expressed during interactions with oomycete and fungal pathogens is enriched for the FORCA promoter motif}

Using Affymetrix microarrays we previously identified clusters of genes that are co-expressed during Arabidopsis immune responses [15]. Two of these clusters, cluster I and the LURP cluster (designated as cluster II in [15]), were defined by distinct patterns of coordinated transcriptional up-regulation during incompatible and compatible interactions with the $H p$ isolates $H p$ Emoy2, HpHiks1 and $H p$ Emco5. While LURP transcripts exhibited a kinetic pattern of late and sustained up-regulation accumulating strongly between 12 and 48 hpi $[16,24]$, cluster I transcript levels tended to increase earlier and predominantly accumulated within the first $12 \mathrm{~h}$ after infection [15]. Genetic disruption of SA-signaling and R protein-mediated $H p$ recognition strongly attenuated or delayed the response of LURP genes, but had only a minor effect on cluster I responses. Inspecting publicly accessible microarray data (Botany Array Resource) [25] we found that cluster I genes also exhibit a pronounced pattern of coactivation during interactions of Arabidopsis with the fungal pathogen Bortytis cinerea as well as a second oomycete, Phythophthora infestans (see Additional File 1). Therefore we renamed cluster I to FORC (Eungal and Oomycete Path-

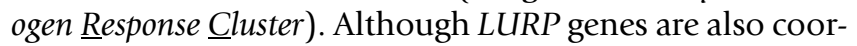
dinately activated in response to the oomycete Phytohthora infestans, this set does not show an uniform response to fungal pathogens (not shown).

Tightly co-expressed subsets of both the FORC and LURP genes were found to be enriched for distinct promoter motifs, such as potential binding sites of WRKY transcription factors [15]. This suggested that members of the FORC cluster on the one hand and the LURP cluster on the other hand are controlled by common regulatory mechanisms. The fact that different motifs are conserved in the promoters of each of these two gene sets suggested that the FORC genes are controlled by mechanisms distinct from those controlling the LURP genes. Applying AlignACE [26] to the $1000 \mathrm{bp}$-upstream sequences of a strictly co-expressed subset of FORC genes (Pearson correlation of 0.95 to the average pattern of the FORC set; [15]) we found the hexameric motif T/ATGGGC to be significantly enriched compared to its statistically expected frequency ( $p=3.0$ E- 4 ; see under "Methods"). We termed this motif $F O R C^{\mathrm{A}}$.

\section{FORCA specifically interacts with a nuclear DNA binding activity that is down-regulated by $\mathrm{Hp}$ and $\mathrm{SA}$}

T/ATGGGC-containing promoter motifs have not been described as pathogen-response elements. Therefore we tested by electrophoretic mobility shift assays (EMSAs), if $F O R C^{A}$ exhibits $H p$-dependent differential interactions with nuclear Arabidopsis proteins (Figure 1). Using the radioactively labeled $F O R C^{\mathrm{A}}-1$ probe consisting of a single copy of the FORCA $C^{A}$ consensus sequence with the TTGGGC core and 8 bp of arbitrary flanking sequence we detected a constitutive DNA-binding activity producing a single band shift that is out-competed by unlabeled FORC ${ }^{\mathrm{A}}-1$ probe (Figures $1 \mathrm{~A}-\mathrm{C}$ ). Surprisingly, this FORC ${ }^{\mathrm{A}}$ binding activity was down-regulated in response to infections with the avirulent $H p$ isolates $H p H i k s 1$ or $H p$ Emoy 2 as well as exogenously applied SA. Furthermore, we found FORCA/ nuclear protein interactions to be light dependent. Nuclear extracts from plants kept for three days under constant light produced substantially more intense FORC ${ }^{\mathrm{A}}$-shifts than those from plants kept for the same time in constant darkness (CL \& CD in Figure 1B). Besides unlabeled $F O R C^{\mathrm{A}}-1$ probe, unlabeled competitor probes containing wild-type FORC ${ }^{A}$ permutations present in several FORC promoters $\left(F O R C^{\mathrm{A}} 2-6\right)$ clearly reduced the intensity of FORCA-1 shifts (Figures 1C \&1D). Mutations in the sequences flanking the invariant TGGGC core of FORC $C_{-}{ }_{-3}$ did not result in reduced competition. However, the FORC $C^{\mathrm{A}}-3$ mutA competitor probe, that lacks the TGGGC core, as well as competitor probes with sequences unrelated to FORCA (M5-2, M8-1) did not efficiently reduce the intensity of FORCA-1 band shifts. These results showed that the observed FORC $C^{\mathrm{A}}$-binding activity is DNAsequence specific and that only the TGGGC sequence of $F O R C^{\mathrm{A}}$ and not sequences flanking this invariant core are critical for this interaction.

\section{FORCA is a light-responsive promoter element whose activity is modulated by defense-related stimuli}

To test effects of $F O R C^{\mathrm{A}}$ on transcriptional regulation, we fused a FORC $C^{\mathrm{A}}$ trimer consisting of three distinct FORC ${ }^{\mathrm{A}}$ permutations to the minimal CaMV35S promoter followed by GUS in pCambia1281X (3xFORC ${ }^{\mathrm{A}}$-pCAMBIA). Untreated Nicotiana benthamiana leaves transiently transformed with $3 \mathrm{xFORC}^{\mathrm{A}}$-pCAMBIA by Agrobacterium tumefaciens exhibited strong GUS activity after histochemical staining or quantitative GUS activity assays (Figure 2A). Treatment of transformed $N$. benthamiana leaves with 1 mM SA clearly reduced this activity. With a construct containing a trimer of a motif unrelated to $F O R C^{\mathrm{A}}(3 \mathrm{xM} 5$ pCAMBIA) we observed no significant reporter gene activ- 


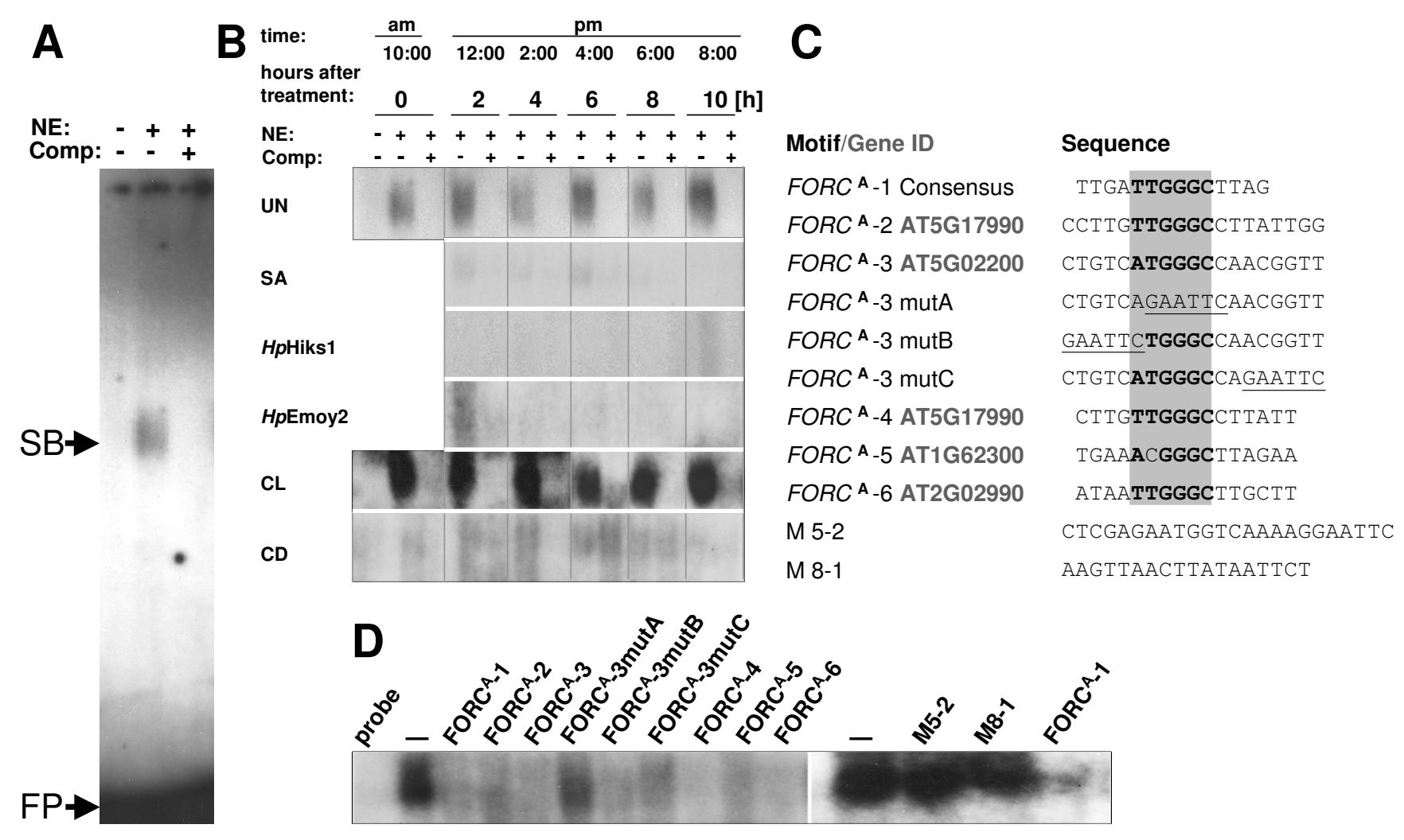

Figure I

In vitro-interactions of FORCA with nuclear Arabidopsis proteins. A, B: Interactions of FORCA with nuclear protein extracts from Arabidopsis Col-0 seedlings grown under normal light conditions and left untreated (UN) or treated with salicylic acid (SA), infected with the avirulent $\mathrm{Hp}$ isolates Hiks I and Emoy2 or exposed for three days to continuous light (CL) or continuous darkness (CD). Panel (A) shows the whole gel for the $0 \mathrm{~h}$ untreated samples; panel (B) shows gel sections with the FORCA-specific band shift for all tested conditions. NE: nuclear extract, Comp: unlabeled competitor probe, SB: FORCA-specific band shift, FP: free probe.C: FORCA derived oligonucleotides used in EMSAs. The gene IDs specify the promoters from which each motif is derived. Positions representing the defining T/ATGGGC core sequences of the shown wild type FORCA permutations are highlighted by a grey box. Sequences mutated in the tested FORCA-3 permutations are underlined. D: EMSA competition analysis of FORCA-I interactions using Arabidopsis nuclear protein extracts from Arabidopsis Col-0 seedlings exposed to CL.

ity, while a trimer of a second unrelated motif, M6, mediated a strong increase of GUS activity upon SA treatment (see Additional File 2). Formally the SA-triggered reduction of $3 \mathrm{xFORC} \mathrm{A}^{\mathrm{A}}$-pCAMBIA expression in our agro-transient assays may be due to effects of SA on the viability of Agrobacterium or other effects unrelated to FORC ${ }^{\mathrm{A}}$-mediated gene expression. However, we believe that this is quite unlikely for the following reasons. (1) we also

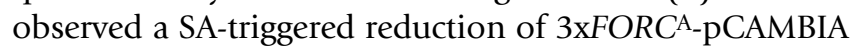
expression in stably transformed Arabidopsis lines (see below); (2) motif M6 mediated SA-inducibility of GUS expression in our agro-transient assays; (3) treatment with $1 \mathrm{mM}$ SA did not reduce GUS expression mediated by the constitutive CaMV35S promoter in previously published agro-transient assays with tobacco leaves $[27,28]$.
pCAMBIA1281X contains a copy of the full CaMV35S promoter close to its multiple cloning site. In order to rule out that this promoter influences the expression pattern observed with $3 \times F O R C^{\mathrm{A}}$-pCAMBIA, we constructed a second pair of reporter gene vectors using pBI101 (Clontech), which lacks any functional promoters in the vicinity of its multiple cloning site. The construct $3 \times F_{O R C}{ }^{\mathrm{A}}$ pBI101 contains a FORCA trimer fused to the minimal CaMV35S promoter followed by GUS. 3xFORC ${ }^{\mathrm{A}}$-mutpBI101 is identical to $3 \times F O R C^{\mathrm{A}}-\mathrm{pBI} 101$, but contains block mutations eliminating the TGGGC core motif of each FORCA copy. Consistent with the results observed with the PCAMBIA versions, $3 \times$ FORC $^{\mathrm{A}}$-pBI101 exhibited in agro-transient expression assays a strong constitutive activity that is suppressed by $\mathrm{SA}$, while $3 \mathrm{xFORC} \mathrm{A}^{\mathrm{A}}$-mut- 

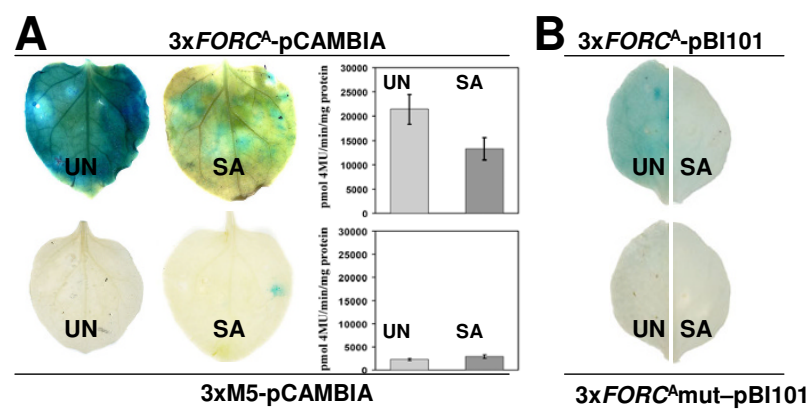

Figure 2

Agrobacterium-mediated transient expression assays in $\mathbf{N}$. benthamiana leaves. A: GUS histochemical staining and specific activity in $N$. benthamiana leaves after a transient expression assay without (UN) or with (SA) a $24 \mathrm{~h}$ salicylic acid treatment using 3xFORCA-pCAMBIA or 3xM5PCAMBIA. Mean and standard error of the quantitative data were calculated from at least six individual measurements. Based on T-tests results for 3xFORCA-pCAMBIA were significantly different $(p=0.026)$, while those for 3xM5-pCAMBIA were not $(p=0.42)$. B: GUS staining in $N$. benthamiana leaves after a transient expression assay without (UN) or with (SA) a $24 \mathrm{~h}$ salicylic acid treatment using $3 \times F_{O R C A}-\mathrm{pBil}$ Ol or 3xFORCAmut-pBilOI.

pBI101 did not show any detectable reporter gene activity (Figure 2B).

We also generated transgenic Arabidopsis lines transformed with $3 \times \mathrm{FORC}^{\mathrm{A}}$-pCAMBIA. T2 progeny from two independent primary transformants were selected for the presence of the transgene and examined for GUS expression by histochemical staining with X-Gluc (Figure 3A). Both tested lines exhibited identical GUS expression patterns. Four day-old 3xFORCA-pCAMBIA seedlings showed constitutive GUS activity in stems which is most pronounced in vascular tissues (Figure 3A-a). Ten day-old seedlings exhibited $3 \mathrm{x} F O R C^{\mathrm{A}}:$ :GUS expression in all plant tissues. In these plants, GUS staining is in particular strongly detectable in vascular tissues and the epidermal tissues surrounding the bases of trichomes (Figure 3A-b). In mature flowers, GUS staining appears in the vascular tissues of sepals and in the anthers, but not in other flower organs (Figure $3 \mathrm{~A}-\mathrm{c}$ ). After fertilization, GUS staining is detected at the top of the flower peduncle (Figure 3A-d). No GUS staining was detectable in any of three tested independent T2 lines containing only the CaMV35 minimal promoter fused to GUS (35Smin-pCambia) (Figure $3 \mathrm{~A}-\mathrm{e}$ ). Consistent with results from our EMSAs (Figure 1), seven day-old $3 \times \mathrm{xFRC}^{\mathrm{A}}$-pCAMBIA plants exhibited stronger GUS accumulation in seedlings grown under CL conditions, compared to either NL- or CD-treated seedlings (Figure $3 \mathrm{~A}-\mathrm{f}, 3 \mathrm{~A}-\mathrm{h}$ and $3 \mathrm{~A}-\mathrm{j}$ ). Application of SA to seedlings grown under NL or CD regime resulted in clearly

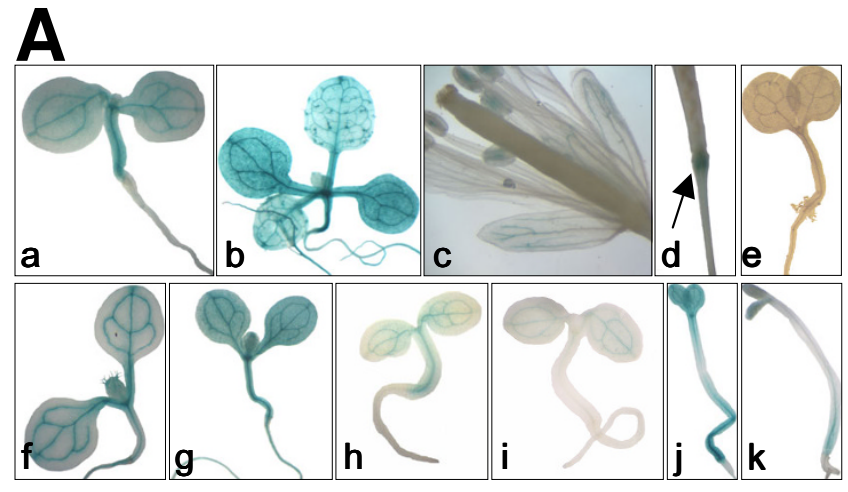

\section{B}

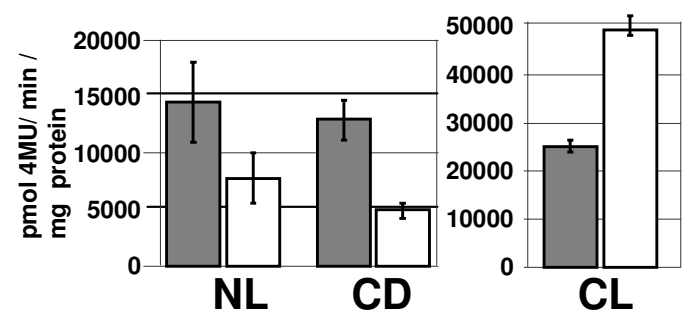

Figure 3

Reporter gene assays in stably transformed Arabidopsis plants. A: Histochemical localization of GUS expression in transgenic Arabidopsis plant carrying 3xFORCA. PCAMBIA. a, 4 day-old seedling; b, 10 day-old plant; c, 30 day-old plant showing GUS staining in pollen grains and sepals; d, Silique showing GUS activity at the top of developing peduncle; e, control 4 day-old seedling carrying the 35Smin-pCAMBIA;f-k, 7 day-old seedlings treated with $0.05 \%$ $\mathrm{EtOH}(\mathrm{f}, \mathrm{h}$ and $\mathrm{j})$ or a $24 \mathrm{~h}$ SA ( $\mathrm{mM}$ ) treatment ( $\mathrm{g}$, i and $\mathrm{k}$ ) under different light conditions: continuous light $(f-g)$, normal light $(\mathrm{h}-\mathrm{i})$, or continuous dark (j-k). B: Fluorometric analysis of GUS specific activities in protein extracts of 3xFORCApCAMBIA plants. Ten day-old plants were treated by either continuous light (CL), normal light (NL) or continuous dark (CD) for three days. After two days light treatment, plants were sprayed with $0.05 \% \mathrm{EtOH}$ (grey bars) or I mM SA (white bars) and shock frozen for protein extraction. Mean and standard error were calculated from six pooled data points generated in three independent experiments, each with two transgenic lines. Based on T-tests all differences between mock and SA-treated samples were significant $(p=$ 9.82E-06 for $\mathrm{CL} ; p=0.038$ for $\mathrm{NL}$ and $p=0.0053$ for $C D$ ). Differences between mock-treated CL and NL samples were also significant $(p=0.0014)$.

reduced reporter gene expression compared to the respective mock-treated controls, (compare Figures 3A-h, 3A-I, $3 \mathrm{~A}-\mathrm{j} \& 3 \mathrm{~A}-\mathrm{k}$ ), while under CL conditions SA treatment resulted in enhanced GUS activity (Figures $3 \mathrm{~A}-\mathrm{f} \& 3 \mathrm{~A}-\mathrm{g}$ ). This effect was particularly clear in tissues outside the vasculature.

Quantitative assays confirmed that in $3 \mathrm{xFORC}^{\mathrm{A}}$-pCAMBIA plants GUS expression is down-regulated by SA under 
normal light conditions as well as after 3 days exposure to constant darkness (Figure 3B). However, after constant light treatment, SA triggered a strong increase of reporter gene expression in these lines. Consistent with the SAinduced suppression of FORCA-mediated GUS expression observed in $3 \times F_{O R C}{ }^{A}$-pCAMBIA Arabidopsis lines under normal light conditions, GUS expression in these lines grown under normal light conditions is also down-regulated after infection with the virulent $\mathrm{Hp}$ isolate Noco2 (Figures 4A, B \&4E). In Col-0 plants, $\mathrm{HpNoco} 2$ is known to induce a basal defense response that is dependent on SA [29].

In our assays the high stability of the GUS enzyme in plant tissue [30] resulted already in strong X-Gluc-staining of $3 \times F^{2} C^{A}$-pCAMBIA seedlings prior to infection with $\mathrm{H} p \mathrm{Noco} 2$ (not shown). To be able to detect a reduction of this background activity, we had to incubate the seedlings for at least 7 days after spray-infection with $\mathrm{HpNoco} 2$ spores. At this timepoint a dense network of $H p$ hyphae had typically developed in cotyledon and leaf tissues. To eliminate the possibility that the reduction of GUS activity in infected tissues is due to disease associated necrosis, we stained $H p$ Noco2-sprayed seedlings 7 dpi with trypan blue, which marks dead plant cells dark blue. As shown in Figure 4D dead plant cells are absent in $\mathrm{H} p \mathrm{NocO} 2$ infected $3 \times F^{2} C^{A}$-pCAMBIA seedlings $7 \mathrm{dpi}$ and only trypan bluestained $H p$ structures are visible in these samples. This
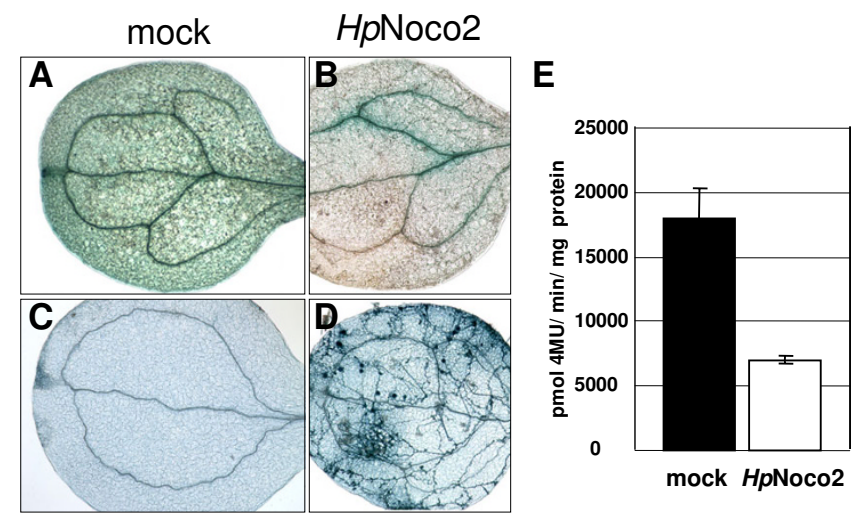

Figure 4

Infections with virulent Hyaloperonospora parasitica suppress FORCA-mediated transcriptional activity. AD: GUS staining (A\&B) of plants treated with $\mathrm{H}_{2} \mathrm{O}(A)$ or $\mathrm{HpNoco} 2$ (B). Trypan blue staining (C\&D) of plants treated with $\mathrm{H}_{2} \mathrm{O}$ (C) or $\mathrm{HpNoco2}(\mathrm{D})$. E: Fluorometric analysis of GUS specific activity in protein extracts of 3xFORCA-pCAMBIA plants subjected to mock-treatment or sprayed with $\mathrm{HpNoco} 2\left(2 * 10^{4}\right.$ spores $\left./ \mathrm{ml}\right)$. Mean and standard error were calculated from four pooled data points generated in two independent experiments, each with two transgenic lines. Based on T-tests the differences were significant $(p=$ 0.0036). indicates that the observed reduction of GUS expression is a result of down-regulated $3 \mathrm{x} F O R C^{\mathrm{A}}$ activity.

Taken together our reporter gene assays using stably transformed Arabidopsis lines, transiently transformed $N$. benthamiana leaves and EMSAs with nuclear Arabidopsis proteins showed that $F O R C^{\mathrm{A}}$ is a constitutive promoter element, the activity of which is modulated by defenseand light-associated signaling processes.

\section{Exposure to constant light enhances basal defense to Hp}

The existence of crosstalk between defense and light signaling suggested that continuous exposure of Arabidopsis seedlings to light affects the efficiency of their defense responses. Indeed, we observed that Col-0 seedlings, when subject to three days of constant light exposure, appear to exhibit reduced susceptibility to $\mathrm{H} p \mathrm{NocO} 2$ (Figure 5). This effect is more pronounced and highly significant in nahG seedlings, which are compromised in defense-associated SA accumulation and, hence, are hyper-susceptible to $\mathrm{H} p \mathrm{NocO} 2$ [31]. In both Col-0 and nahG seedlings constant light pre-treatment resulted in the same level of protection to $\mathrm{HpNocO} 2$, indicating that this effect does not require SA.

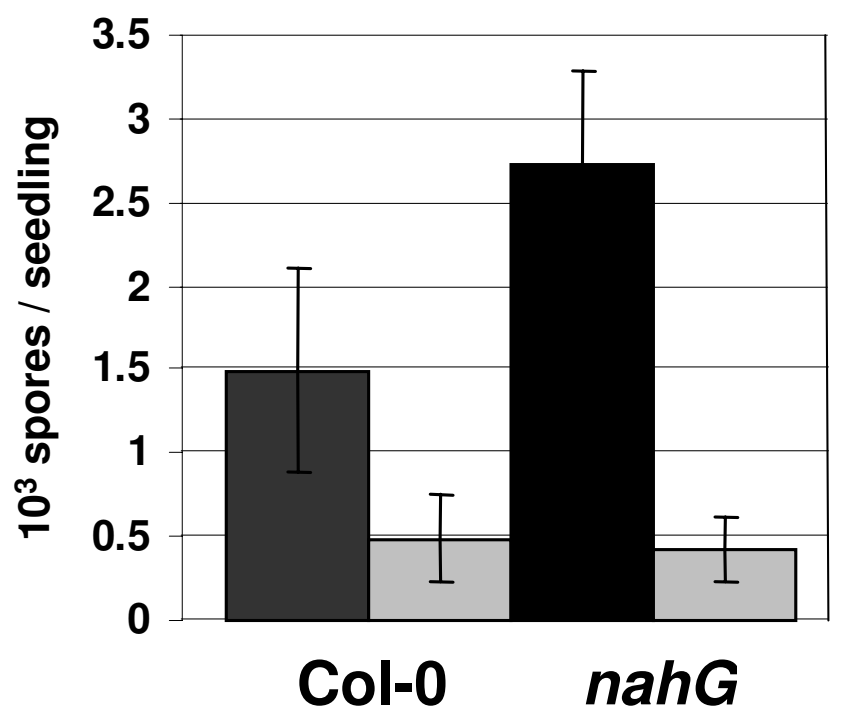

Figure 5

Continuous exposure to light results in reduced susceptibility to virulent Hyaloperonospora parasitica. $\mathrm{HpNoc02}$ growth has been evaluated on plants pretreated for 3 days by normal light (dark grey) or continuous light (light grey) exposure. Two weeks old plants were sprayed with HpNoco2 $\left(2 * 10^{4}\right.$ spores $\left./ \mathrm{ml}\right)$ and spores were counted $7 \mathrm{dpi}$. Bars represent mean value of spores/seedling from four different experiments. Based on T-tests differences between normal light and constant light treated samples were significant only for nahG plants $(p=0.0084)$. 


\section{Discussion}

Both PTI and ETI are associated with massive transcriptional reprogramming [19,32]. Although multiple types of cis-elements and transcription factors controlling defense-related genes have been identified, the important regulatory step of activation of the plant defense transcriptome is still poorly understood. Our current knowledge about defense-associated transcriptional regulation has mainly resulted from the analysis of individual "model genes" such as the Arabidopsis PR1 and Parsley PR10 genes $[33,34]$. The vast amount of global transcript profiling data that has accumulated for Arabidopsis and other model plants now allows us to address the identification of such regulatory mechanisms from a new perspective enabling researchers to directly pursue the discovery of transcriptome-level control elements. Multiple studies reported the existence of statistically conserved promoter motifs in clusters of co-expressed genes [20,21,35], but only a few reports provided evidence for their roles as regulatory promoter elements as well as binding sites of nuclear proteins $[22,23]$.

Here we provide another successful demonstration supporting the feasibility of this approach. FORC ${ }^{\mathrm{A}}$-related motifs have not been implicated in the control of defenseassociated gene expression before. We found FORCA to DNA-sequence specifically interact with nuclear Arabidopsis proteins. These interactions are differential and suppressed by defense-related stimuli. Furthermore, our data showed FORCA to function as a constitutive promoter element, whose activity can be modulated by the defense hormone SA or recognition of $H p$.

The FORC cluster constitutes a set of genes that are coordinately up-regulated in response to infections by oomycete and fungal pathogens. In addition, 19 of the 43 FORC genes represented on the Affymetrix ATH1 array are significantly up-regulated by the SA analog BTH [36]. As in Arabidopsis plants grown under a normal light regime the constitutive FORC $C^{\mathrm{A}}$ activity is suppressed by defense-stimuli, including SA, this element cannot be sufficient to control the defense-associated expression of FORC genes. The FORC-type expression pattern can only be the result of the combined activity of FORC ${ }^{\mathrm{A}}$ and additional cis-elements that act as positive pathogen response elements. We previously reported the enrichment of a W box-like motif and an unknown motif in the promoters of FORC genes, which may mediate the pathogen-induced up-regulation of this cluster [15]. Indeed, we found these motifs to exhibit $H p$-inducible band shifts in EMSAs with nuclear Arabidopsis proteins (not shown), suggesting a positive contribution to transcriptional activity. The role of $F O R C^{\mathrm{A}}$ may be to limit the amplitude of FORC gene induction and/or to mediate the down-regulation of FORCs after an early peak of their transcript levels is reached. Future experiments will have to address this possibly interesting interplay between different pathogen-response elements.

Both defense-related and light-related stimuli affect the transcriptional output mediated by FORCA ${ }^{\mathrm{A}}$. Under normal light conditions or constant darkness FORC ${ }^{\mathrm{A}}$ exhibits a constitutive activity that is suppressed by defense-related stimuli. Under constant light-exposure $F O R C^{\mathrm{A}}$ acts as a positive defense-related element mediating SA-inducible gene expression. The latter feature of FORCA, however, is not reflected in our EMSAs with nuclear Arabidopsis proteins. Under constant light conditions no further increase of the intensity of FORC $C^{\mathrm{A}}$ band shifts was observed after SA treatment (not shown). This may indicate that under constant light conditions FORC ${ }^{\mathrm{A}}$-dependent pathogen inducibility is not mediated by enhanced binding of nuclear factors, but rather other mechanisms that cannot be detected under our EMSA conditions, such as subtle post-translational modifications of pre-bound factors or transient interactions with co-factors.

To our knowledge FORCA is unrelated to any described defense-associated promoter elements. Using plant ciselement databases (Plant CARE and PLACE, http://bioin formatics.psb.ugent.be/webtools/plantcare/html/; http:// www.dna.affrc.go.jp/PLACE/), however, we found several known FORCA-like promoter motifs that have been implicated in other biological processes (Figure 6). The bean stem element 1 (SE1), that appears to act as a strong unspecific enhancer, and the oat light-repressor element 1 (RE1), which suppresses light-driven reporter gene expression, contain sequences that perfectly match the FORCA consensus $[37,38]$. The positive light response element PE1 from oat contains a sequence that matches 5 of the 6 conserved positions of FORCA [38]. RE1 and PE1 are conserved in the promoters of multiple phyA genes that are regulated in a light-dependent manner. Inspecting a data set on light triggered transcriptome changes in Arabidopsis [39] we also found $F O R C^{A}$ to be conserved in the promoters of phytochrome A\&B-dependently light-induced genes encoding proteins related to photosynthesis or localized to the chloroplast. In these 26 promoters the $F O R C^{\mathrm{A}}$ hexamer is significantly enriched compared to its statistically expected frequency ( $p=5.82 \mathrm{E}-9)$. If FORCA contributes to the co-regulation of these genes remains to be shown. In addition, a motif that perfectly matches the FORC ${ }^{\mathrm{A}}$ consensus was found to be conserved in Arabidopsis core promoters [40]. A function has not been assigned to this motif, but its preferential location at the distal periphery of core promoter regions may suggest a role distinct from that of conventional core promoter elements. $F O R C^{\mathrm{A}}$ does also perfectly match the recently identified "protein box" (PBX) which confers phase-specific diurnal and circadian reporter gene expression in Arabidopsis 


\begin{tabular}{|c|c|c|c|}
\hline \multirow{10}{*}{ Oat } & em element 1 (SE1): & AATGGG & (Keller \& Baumga \\
\hline & or element 1 (REI): & CATGGGCGCGG & (Bruce et al.; 1991) \\
\hline & Rice RE1-like: & GATGGGGGAAG & (Bruce et al.; 1991) \\
\hline & Maize RE1-like: & CATGGGCACCG & (Bruce et al.; 1991) \\
\hline & Oat PE3 a: & GATGGGAGAGC & (Bruce et al.; 1991) \\
\hline & Oat PE3 b: & CATGGGAGCTG & (Bruce et al.; 1991) \\
\hline & Rice PE3-like a: & GATGGGGTCGC & (Bruce et al.; 1991) \\
\hline & Rice PE3-like b: & GTGGGGAGGTG & (Bruce et al.; 1991) \\
\hline & Maize PE3-like a: & CGTGGGCCGCG & (Bruce et al.; 1991) \\
\hline & Maize PE3-like b: & GGCGGGAGGTG & (Bruce et al.; 1991) \\
\hline \multirow[t]{4}{*}{ Arabidopsis } & core prom. motif 7 : & TTGGGCTT & (Molina \& Grotewold; 2005) \\
\hline & & & \\
\hline & $\begin{array}{l}\text { Arabidopsis PBX: } \\
\text { FORC Consensus: }\end{array}$ & $\begin{array}{l}\text { ATGGGCC } \\
\text { TTGGGC }\end{array}$ & (Micheal et al., 2008) \\
\hline & & A & \\
\hline
\end{tabular}

Figure 6

FORCA is related to multiple described promoter elements. Known or hypothetical promoter elements with sequence similarities to FORCA are aligned. Positions matching the FORCA consensus motif are highlighted in grey.

[41]. Promoters that contain PBX are enriched for gene ontology annotations related to protein synthesis.

Taken together, these reports suggest that FORCA/PBXrelated cis-elements are conserved throughout the plant kingdom and are generally involved in adjusting the transcriptome to daily changes in light-related (and possibly other) environmental conditions. Furthermore, these ciselements appear to participate in the regulation of a diverse set of genes involved in crucial metabolic processes such as photosynthesis and protein biosynthesis, but also defense. Consistent with such a broad role we observed a 2.9-fold higher frequency of the FORC ${ }^{\mathrm{A}}$ hexamer in Arabidopsis intergenic sequences than statistically expected. On average, FORCA is present 0.78 times per 1 $\mathrm{kb}$ in all $1 \mathrm{~kb}$ sequences upstream from the 33,282 loci of the TAIR8 Arabidopsis genome annotation http:// www.Arabidopsis.org, while only 0.27 occurrences of this hexamer per $1 \mathrm{~kb}$ are statistically expected in Arabidopsis intergenic regions.

It is presently unclear, what biological purpose it serves that defense-related stimuli reduce FORC ${ }^{\mathrm{A}}$ activity under normal light conditions or constant darkness, but enhance its activity under constant light exposure. This seemingly unorthodox and contradictory feature of $F O R C^{\mathrm{A}}$ may reflect that in natural environments the probability of plant/pathogen encounters can follow complex diurnal patterns which are further influenced by transient environmental factors, such as local light conditions, temperature, humidity and the presence of leaf surface water [42]. Hence, FORC $C^{A}$ may act in concert with other regulatory elements to generate fine-tuned gene expression outputs appropriate for a changing array of environmental conditions.

Crosstalk between light and defense signaling has been described in several earlier reports and high light fluence rates or long durations of light exposure have been positively correlated with plant defense reactions $[12,13]$. For example, Genoud et al. reported interactions between phytochrome-mediated light responses and SA-triggered responses in Arabidopsis [12]. SA-dependent responses such as expression of the defense marker PR1 and resistance to avirulent Pseudomonas syringae bacteria were found to be triggered by exposure to high fluence rates of white light and to require a functional phytochrome signaling apparatus. It is unclear, however, if the functional characteristics of FORCA that we observed are related to the effects described by Gernoud et al., as different lightrelated parameters were tested in each study. While Gernound et al. varied the fluence rate of white light, we varied the length of the photoperiod. Multiple lesion mimic mutants also point to interactions between light and defense signaling, as they are known to exhibit spontaneous defense responses, such as elevated $P R$ gene expression, upon exposure to high light $[43,44]$. As a possible connection between light and defense signaling 
in these mutants, light-driven accumulation of reactive oxygen intermediates (ROI), such as superoxide and hydrogenperoxide has been discussed $[42,45]$. ROI act as an early defense signal and participate in feedback mechanisms modulating SA-dependent defense responses $[46,47]$.

Future experiments will have to address what types of light- and defense signals are important for the crosstalk perceived by FORCA and if FORCA-mediated responses are responsible for the reduced susceptibility to $\mathrm{HpNoco} 2$ we observed in Arabidopsis seedlings subject to constant light exposure. A number of FORC members appear to show enhanced transcript levels after exposure to a prolonged photoperiod or high light intensity (see Additional File 3). Although this would be consistent with a role of $F O R C^{\mathrm{A}}$ in light-triggered defense responses, further experimentation will be needed to establish such a functional connection. Moreover, it remains to be examined, if the interference between defense and light signaling we have observed is manifested in signal convergence directly at $F O R C^{\mathrm{A}}$ and its cognate transcription factors or in regulatory steps operating further upstream. Alternatively, such crosstalk may not be mediated by discrete signaling steps at all and may be merely a consequence of general disturbances of a highly complex plant signaling network [48]. At this point our knowledge about the architecture and dynamics of regulatory networks in plants is only rudimentary. Emerging new methodologies of computational biology, functional genomics and systems biology will allow us to shift the focus from understanding individual signaling circuits to a more holistic view of regulatory processes. Having promoter elements at hand that integrate signals from multiple input channels will aid in the dissection of such complex regulatory interactions.

\section{Conclusion}

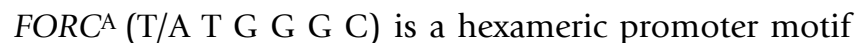
that is conserved in clusters of Arabidopsis genes coexpressed in response to fungal or oomycete pathogens as well as defined light treatments. It constitutively interacts in a DNA-sequence specific manner with nuclear Arabidopsis proteins. These interactions are suppressed by defense-related stimuli and enhanced by prolonged exposure to constant light. Furthermore FORC ${ }^{\mathrm{A}}$ mediates constitutive reporter gene expression in transiently transformed $N$. benthamiana leaves as well as in stably transformed Arabidopsis plants. Its responsiveness to defense-stimuli is modulated by the duration of light exposure. We propose that FORCA integrates light and defense related signals and participates in adjusting the transcriptome to daily changes in light-related environmental conditions.

\section{Methods \\ Plants material and treatments}

Arabidopsis ecotype Col-0, transgenic Col-0 nahG plants [31] and Nicotiana benthamiana plants were grown on soil under fluorescent lights $\left(21^{\circ} \mathrm{C}, 100 \mathrm{E} \mathrm{m}^{-2} \mathrm{sec}^{-1}\right)$. Normal light cycles were $14 \mathrm{~h}$ day/10 h night. For some experiments, seedlings grown under normal conditions were exposed for additional three days to continuous light or dark treatment. Hyaloperonospora parasitica $(H p)$ was grown, propagated and applied to Arabidopsis as previously described [49]. Two week-old seedlings were sprayinoculated with $H p$ spore suspensions $\left(6 \times 10^{4}\right.$ to $1 \times 10^{5}$ spores $\mathrm{ml}^{-1}$ of water for $H p H i k s 1$ and $H p$ Emoy2 and $2 \times$ $10^{4}$ spores $\mathrm{ml}^{-1}$ for $\mathrm{H} p \mathrm{Noco} 2$ ) with Preval paint sprayers. $H p H i k s 1, H p$ Emoy 2 and $H p N o c o 2$ growth was determined 7 dpi by trypan blue tissue staining or visual sporangiophore counts [49]. SA was applied to Arabidopsis and $N$. benthamiana at a concentration of $1 \mathrm{mM}$ in $0.05 \%$ EtOH using Preval sprayers.

\section{Preparation of nuclear proteins and electrophoretic mobility shift assays}

Nuclear protein extractions were performed as previously described [50] from $15 \mathrm{~g}$ of Arabidopsis whole seedlings that were untreated or pre-treated as described in the legend of Figure 1. Bio-Rad Bradford assays were used to determine protein concentrations. EMSAs were performed using the synthetic oligonucleotides (invitrogen) listed in Figure 1C. Fifty picomoles of double-stranded oligonucleotides were radio-labeled using $\mathrm{T} 4$ polynucleotide kinase (NEB) and $\gamma^{32}$ P-ATP. 2.5 picomole G50-purified radioactively labeled double stranded probe (10000 to 30000 $\mathrm{cpm})$ was incubated with $15 \mu \mathrm{g}$ of nuclear protein in a total volume of $30 \mu \mathrm{l}$ of binding buffer $(20 \mathrm{mM}$ Hepes$\mathrm{KOH} \mathrm{pH} \mathrm{7.9;} 1.5 \mathrm{mM} \mathrm{MgCl}_{2} ; 0.2 \mathrm{mM}$ EDTA; $200 \mathrm{mM}$ $\mathrm{NaCl}$; $1 \mathrm{mM}$ DTT) for $20 \mathrm{~min}$ at room temperature. For competition experiments, 100 -fold molar excess of unlabeled competitor was added to the binding reaction. The EMSA reactions were subjected to electrophoresis on $5.4 \%$ non-denaturing polyacrylamide gels in $0.5 \times$ tris borate EDTA buffer (TBE) at room temperature. The gels were vacuum dried and autoradiographed for 5 days at $-80^{\circ} \mathrm{C}$ on HyBlot CL autoradiography film (Denville Scientific).

\section{Molecular cloning \\ Trimers consisting of three different FORCA permutations (5'-TACGCCGAATTCAAGCTTCTTGTTGGGCCTTAAAA ACAATTGGGCAATCAAAAACATTGGGCAGTGTCTAGA CTCGAGGATTACGCC-3') or three different permuta- tions of the M5 motif (5'-TACGCCG AATTCAAGCT- TCTTTTGAC CATTCTTTTGACCATTCTTTTGACCATTCTC GAGGTCGACGATTACGCC-3') as well as the M6 motif (5'TTGATATCGAATTCCTAAGTGAAGAAGAAACGAGCA TCTTAAGAAGAAGTCGTGACAAAAAAGAAGAAGATCA}


GGTCTCGAGGTCGACGG-3') were cloned as HindIIISalI inserts into pBT10 [51]. The resulting $3 \mathrm{xFORC}^{\mathrm{A}_{-}}$ pBT10 and 3xM5-pBT10, which include the -46 bp CaMV35S minimal promoter (35Smin) present in pBT10 in addition to the respective trimeric promoter motif, were digested with NcoI and HindIII. These HindIII-NcoI inserts were fused to the GUS reporter gene in plasmid pCambia1281X to give $3 \times F_{O R C}{ }^{\mathrm{A}}$-pCAMBIA, $3 \mathrm{xM} 5$ pCAMBIA and 3xM6-pCAMBIA. 3xFORC ${ }^{\mathrm{A}}$-pCAMBIA was digested with HindIII and SalI. After purification the linearized 3xFORC ${ }^{\mathrm{A}}$-pCAMBIA was blunt-ended and ligated to give 35Smin-pCambia as a negative control plasmid. $3 \mathrm{x} F O R C^{\mathrm{A}}$-pCAMBIA was PCR amplified with pBI-HindIIIF (5'-GATTACGCCAAGCTTGAATTC-3') and pBI-Xba-R (5'-GAAATTTACCTCTAGATCTACC-3'). The PCR product was digested by HindIII and $\mathrm{XbaI}$ and introduced into pBi101.1 (Clontech) to give $3 \mathrm{xFORC}^{\mathrm{A}}$-pBi101. $3 \mathrm{x} F O R C^{\mathrm{A}_{-}}$ pBi101 was digested with HindIII and SalI to remove the $3 \times F$ ORC ${ }^{\mathrm{A}}$ sequence. The trimer of FORC $\mathrm{A}_{-}$ mut(5'TACGCCGAATTAAGCTTCCTTGGAATT CCTTAAA AACAAGAATTCAATCAAAAACAGAATTCAGTGTCTAGA GTCGACGATTACGCC-3') was digested with HindIII and SalI, and introduced into $3 \times F_{O R C} \mathrm{~A}_{-}$pBi101 which was predigested by HindIII and SalI to give $3 \mathrm{xFORC}^{\mathrm{A}}$-mutpBi101. All restriction digestion products used for cloning (Trimers, PCR products and plasmids) were purified with the QIAquick PCR purification kit (Qiagen). All plasmids have been propagated using Escherichia coli DH5alpha. The sequences of inserts and fusion sites with 35Smin and the GUS reporter gene in pCambia1281X and pBi101.1 vectors were verified by sequencing for $3 \mathrm{xFORC}^{\mathrm{A}}$ - $\mathrm{pBT} 10$, 3xM5-pBT10, 3xFORCA-pCAMBIA， 3xM5-pCAMBIA,

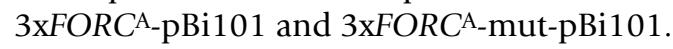

\section{N. benthamiana transient expression assays}

Transient expression of 3xFORCA-pCAMBIA, 3xM5pCAMBIA in Nicotiana benthamiana was based on the protocol established by Popescu et al. [52]. Plasmids 3xFORCA ${ }^{\mathrm{A}}$-pCAMBIA, 3xM5-pCAMBIA, 3xM6-pCAMBIA, $3 \times F_{R} C^{A}-p B i 101,3 \times F^{2} C^{A}-m u t-p B i 101$ and p19 (a viral RNA silencing suppressor from the tomato bushy stunt virus) were first transformed into A. tumefaciens GV3101 by electroporation. One transformed colony (verified by PCR) for each plasmid was cultured at $28^{\circ} \mathrm{C}$ until the $A_{600}$ reached 1 to 1.5 . The bacteria were centrifuged for $15 \mathrm{~min}$ at $2,000 \mathrm{~g}$, resuspended in infiltration

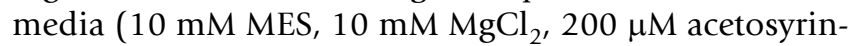
gone) to a final $A_{600}$ of 1.5 , and incubated for 3 to $4 \mathrm{~h}$ at room temperature. Bacteria were then washed with 1 volume of fresh infiltration media, and resuspended as described above. A mix of a 1:1 ratio (final OD of 0.75 ) between Agrobacteria containing 3xFORC $C^{\mathrm{A}}$-pCAMBIA, 3xM5-pCAMBIA， 3xM6-pCAMBIA， 3xFORC ${ }^{\mathrm{A}}$-pBi101,
$3 \mathrm{xFORC} \mathrm{A}^{\mathrm{A}}$-mut-pBi101 and those containing p19 were used for infiltration. The infiltration of bacterial mixtures was performed with a 1-ml syringe on the abaxial side of leaves of 3-week-old $N$. benthamiana plants. After 48 h, mock (EtOH $0.05 \%$ ) or SA treatments were applied and leaves either shock frozen in liquid nitrogen or $\mathrm{x}$ gluc stained (see below).

\section{Generation of transgenic Arabidopsis lines}

35 Smin-pCambia and $3 \times F^{2}{ }^{\mathrm{A}}$-pCAMBIA was introduced in the A. tumefaciens strain AGLO2 by electroporation [53]. Agrobacterium-mediated transformation of Col-0 $\left(\mathrm{T}_{0}\right)$ was performed by the floral-dip method [54]. 35 Smin-pCambia and $3 \times F O R C^{\mathrm{A}}$-pCAMBIA transgenic plants were selected on $0.5 \mathrm{MS} / .0 .8 \%$ agar media containing $50 \mathrm{mg} \mathrm{l}^{-1}$ hygromycinin in the dark for 1 week and then transferred to soil. T2 lines were used for GUS staining and fluorometric analysis.

\section{GUS histochemical staining}

For N. benthamiana transient assays, transformed leaves were infiltrated as described above with a solution containing $1 \mathrm{mg} \mathrm{ml}^{-1}$ 5-bromo-4-chloro-3-indoyl- $\beta$-d-glucuronide (X-Gluc), $50 \mathrm{~mm} \mathrm{Na} \mathrm{PO}_{4} \mathrm{pH} 7.2,0.5 \mathrm{~mm}$ $\mathrm{K}_{3} \mathrm{Fe}(\mathrm{CN})_{6}, 0.5 \mathrm{~mm} \mathrm{~K}_{4} \mathrm{Fe}(\mathrm{CN})_{6}$ incubated $\mathrm{O} / \mathrm{N}$ at $37^{\circ} \mathrm{C}$ and cleared with $70 \%$ ethanol (EtOH). For Arabidopsis transgenic plants, whole seedlings were vacuum infiltrated for $15 \mathrm{~min}$, incubated $\mathrm{O} / \mathrm{N}$ at $37^{\circ} \mathrm{C}$ and cleared with $70 \%$ EtOH.

\section{Fluorometric analysis of GUS activity}

Protein was extracted from 15-20 Arabidopsis seedlings or 5-6 N. benthamiana leaves in MUG extraction buffer (50 mm sodium phosphate ( $\mathrm{pH} 7.0), 10 \mathrm{~mm}$ EDTA ( $\mathrm{pH}$ $8.0), 0.1 \%$ SDS and $0.1 \%$ Triton X-100). Ten microliters of crude extract was added to $290 \mu \mathrm{l}$ of reaction mix containing $10 \mathrm{mM}$ 4-MUG (Gold Biotechnology, St. Louis, $\mathrm{MO}$ ) in MUG extraction buffer. Reactions were stopped at 15-min intervals by adding $50 \mu \mathrm{l}$ of MUG reaction mixture to $200 \mu \mathrm{l} 1 \mathrm{M}$ sodium carbonate. Reactions were performed in 96 -well plates at $37^{\circ} \mathrm{C}$. Fluorescence was measured at an excitation wavelength of $365 \mathrm{~nm}$ and emission wavelength $455 \mathrm{~nm}$ using Wallac 1420-012 Multilabel Counter (PerkinElmer Life Science). Protein concentrations in extracts were determined using BioRad protein assays following the manufacturer's instructions (Bio-Rad Laboratories). The GUS activity was expressed as picomoles of 4-methyl umbelliferone per milligram of protein per minute. Final values were adjusted to eliminate background fluorescence by subtracting the average GUS activity measured from non- $\beta$ glucuronidase (uidA, GUS) expressing Arabidopsis seedlings. For $N$. benthamiana, GUS activity values are 
expressed as means of 5-10 samples each containing 56 leaves for three experimental repetitions. For GUS expressing transgenic Arabidopsis lines GUS activity values are means of 5-10 samples each containing 15-20 seedlings from two independent lines for three independent experimental repetitions.

\section{Statistical analyses of conserved promoter motifs}

$P$-values describing the enrichment of defined sequence motifs in promoter sets were calculated using the Poisson distribution as described previously [15]. However, due to the general conservation of FORC $C^{\mathrm{A}}$ in a large number of Arabidopsis promoters, the expected frequency of this motif was calculated based on theoretical assumptions and not based on its actual occurrence in all Arabidopsis promoters as previously [15]. Arabidopsis intergenic regions contain approximately 16\% "Cs", 16\% "Gs", $32 \% "$ As" and 32\% "Ts". Based on this the expected frequency of the FORC ${ }^{A}$ hexamer (A/T T G G G C) approximately equals $0.64 \times 0.32 \times 0.16^{4} \times 2 \times 1000=0.27$ occurrences on both strands of $1 \mathrm{~kb}$ of random intergenic DNA.

\section{Authors' contributions}

AE designed and performed all experiments and wrote parts of the manuscript. NT performed the statistical analyses. TE supervised this project and wrote parts of this manuscript.

\section{Additional material}

\section{Additional file 1}

Arabidopsis FORC genes are co-expressed in response to infections with the oomycete Phytphthora infestans and the necrotrophic fungus Botrytis cinerea. Represented are transcript levels triggered in Arabidopsis (accession Col-0) infected by Phytophthora infestans (Pi) or Botrytis cinerea (Bc). Transcript levels are illustrated as ratios between infected and mock treated control samples (red signal signifies an up-regulation in infected tissue relative to the control). All transcript data were generated using Affymetrix ATH1 whole genome arrays and were downloaded in analyzed form [25] from the Botany Array Resource web site http://bbc.botany.utoronto.cal. They were provided by Dierk Scheel, Frederic Brunner \& Lore Westphal (Pi) as well as Carine Denoux, Fred Ausubel, Julia Dewdney \& Simone Ferrari (Bc).

Click here for file

[http://www.biomedcentral.com/content/supplementary/14712229-9-2-S1.ppt]

\section{Additional file 2}

Agrobacterium-mediated transient expression assays with 3xM6pCAMBIA in N. benthamiana leaves. GUS staining in N. benthamiana leaves after a transient expression assay without (UN) or with (SA) a $24 \mathrm{~h}$ salicylic acid treatment using 3xM6-pCAMBIA. Shown are typical examples of $\mathrm{N}$. benthamiana leaves.

Click here for file

[http://www.biomedcentral.com/content/supplementary/14712229-9-2-S2.ppt]

\section{Additional file 3}

Transcript levels of Arabidopsis FORC genes are affected by photoperiod and light intensity. Represented are relative transcript levels of FORC genes after exposure of Arabidopsis plants to different photoperiods or 3 h-exposure to a high fluence rate of $1000 \mathrm{uM} \mathrm{m}{ }^{-2} s^{-1}$ white light (in Col-0 wild type, cry1 or hy5 mutant plants). All transcript data were generated using Affymetrix ATH1 whole genome arrays and were downloaded in analyzed form [55] from the Genevestigator web site https:// www.genevestigator.ethz.ch/gv/index.jsp. Transcript levels are illustrated as ratios between plants exposed to an $8 \mathrm{~h}$ photoperiod and plants exposed to a $16 \mathrm{~h}$ photoperiod (Photoperiod $8 \mathrm{~h} / 16 \mathrm{~h}$ ) or as ratios between plants exposed to high white light fluence rates versus control treated plants (high light). The scale bar on the left hand side relates color intensity to linear fold-change values. For the "photoperiod data set" green signal signifies an up-regulation in plants exposed to the longer photoperiod relative to plants exposed to the shorter photoperiod. For the "high fluence rate data set" red signal signifies an up-regulation triggered by high light intensity. The data were generated by M. Schmid and D. Weigel (photoperiod) and Kleine et al. [56] (high fluence rate).

Click here for file

[http://www.biomedcentral.com/content/supplementary/1471-

2229-9-2-S3.ppt]

\section{Acknowledgements}

This work was supported by NSF-IOB grant \# 0449439 to TE. We thank Linda Saetern and Noelle Oas for excellent technical assistance and YuHung (Linda) Wei (UC-Riverside) for critical reading of the manuscript.

\section{References}

I. Nürnberger T, Brunner F, Kemmerling B, Piater L: Innate immunity in plants and animals: striking similarities and obvious differences. Immunol Rev 2004, I 98:249-266.

2. Chisholm ST, Coaker G, Day B, Staskawicz BJ: Host-microbe interactions: shaping the evolution of the plant immune response. Cell 2006, I 24(4):803-814

3. Jones JD, Dangl JL: The plant immune system. Nature 2006, 444(7 I I 7):323-329.

4. Chang JH, Goel AK, Grant SR, Dangl JL: Wake of the flood: ascribing functions to the wave of type III effector proteins of phytopathogenic bacteria. Curr Opin Microbiol 2004, 7(I): I I- I8.

5. Klessig DF, Durner J, Noad R, Navarre DA, Wendehenne D, Kumar D, Zhou JM, Shah J, Zhang S, Kachroo P, Trifa Y, Pontier D, Lam E, Silva $H$ : Nitric oxide and salicylic acid signaling in plant defense. Proc Natl Acad Sci USA 2000, 97( ( 6):8849-8855.

6. Nimchuk Z, Eulgem T, Holt IB, Dangl JL: Recognition and response in the plant immune system. Annu Rev Genet 2003, 37:579-609.

7. Glazebrook J: Contrasting mechanisms of defense against biotrophic and necrotrophic pathogens. Annu Rev Phytopathol 2005, 43:205-227.

8. Mur LA, Kenton P, Atzorn R, Miersch O, Wasternack C: The outcomes of concentration-specific interactions between salicylate and jasmonate signaling include synergy, antagonism, and oxidative stress leading to cell death. Plant Physiol 2006, I 40(I):249-262.

9. Spoel SH, Koornneef A, Claessens SM, Korzelius JP, Van Pelt JA, Mueller MJ, Buchala AJ, Metraux JP, Brown R, Kazan K, Van Loon LC, Dong $X$, Pieterse CM: NPRI modulates cross-talk between salicylate- and jasmonate-dependent defense pathways through a novel function in the cytosol. Plant Cell 2003, I5(3):760-770.

10. Glazebrook J, Chen W, Estes B, Chang HS, Nawrath C, Metraux JP, Zhu T, Katagiri F: Topology of the network integrating salicylate and jasmonate signal transduction derived from global expression phenotyping. Plant J 2003, 34(2):21 7-228. 
II. Robert-Seilaniantz A, Navarro L, Bari R, Jones JD: Pathological hormone imbalances. Curr Opin Plant Biol 2007, 10(4):372-379.

12. Genoud T, Buchala AJ, Chua NH, Metraux JP: Phytochrome signalling modulates the SA-perceptive pathway in Arabidopsis. Plant J 2002, 3 I (I):87-95.

13. Griebel T, Zeier J: Light regulation and daytime dependency of inducible plant defenses in Arabidopsis: phytochrome signaling controls systemic acquired resistance rather than local defense. Plant Physiol 2008, 147(2):790-80I.

14. Logemann E, Hahlbrock K: Crosstalk among stress responses in plants: pathogen defense overrides UV protection through an inversely regulated ACE/ACE type of light-responsive gene promoter unit. Proc Natl Acad Sci USA 2002, 99(4):2428-2432.

15. Eulgem T, Weigman V], Chang H-S, McDowell JM, Holub EB, Glazebrook J, Zhu Ta, Dangl JL: Gene Expression Signatures from Three Genetically Separable Resistance Gene Signaling Pathways for Downy Mildew Resistance. Plant Physiology 2004, 135: I I29-1 I 44

16. Knoth C, Ringler J, Dangl JL, Eulgem T: Arabidopsis WRKY70 is required for full RPP4-mediated disease resistance and basal defense against Hyaloperonospora parasitica. Molecular Plant Microbe Interactions 2007, 20(2): $120-128$.

17. Knoth $C$, Eulgem $T$ : The oomycete response gene LURPI is required for defense against Hyaloperonospora parasitica in Arabidopsis thaliana. The Plant Journal 2008, 55:53-64.

18. Dong X: NPRI, all things considered. Curr Opin Plant Biol 2004, 7(5):547-552.

19. Eulgem T: Regulation of the Arabidopsis defense transcriptome. Trends Plant Sci 2005, I0(2):7I-78.

20. Maleck K, Levine A, Eulgem T, Morgan A, Schmid J, Lawton KA, Dangl JL, Dietrich RA: The transcriptome of Arabidopsis thaliana during systemic acquired resistance. Nat Genet 2000, 26(4):403-4I0.

21. Chen W, Provart NJ, Glazebrook J, Katagiri F, Chang HS, Eulgem T, Mauch F, Luan S, Zou G, Whitham SA, Budworth PR, Tao Y, Xie Z, Chen X, Lam S, Kreps JA, Harper JF, Si-Ammour A, Mauch-Mani B, Heinlein M, Kobayashi K, Hohn T, Dangl JL, Wang X, Zhu T: Expression profile matrix of Arabidopsis transcription factor genes suggests their putative functions in response to environmental stresses. Plant Cell 2002, I 4(3):559-574.

22. Harmer SL, Hogenesch JB, Straume M, Chang H-S, Han B, Zhu T, Wang X, Kreps JA, Kay SA: Orchestrated transcription of key pathways in Arabidopsis by the circadian clock. Science 2000 , 290:2110-2113.

23. Wang D, Weaver ND, Kesarwani M, Dong X: Induction of protein secretory pathway is required for systemic acquired resistance. Science 2005, 308(5724): I036-1040.

24. Eulgem T, Tsuchiya T, Wang X, Cuzick A, Beasley B, Toer M, McDowell JM, Holub E, Zhu T, Dangl JL: EDM2 is required for RPP7-dependent disease resistance in Arabidopsis and affects RPP7 transcript levels. The Plant Journal 2007, 49:829-839.

25. Toufighi K, Brady SM, Austin R, Ly E, Provart NJ: The Botany Array Resource: e-Northerns, Expression Angling, and promoter analyses. Plant J 2005, 43(I): 153-163.

26. Hughes JD, Estep PW, Tavazoie S, Church GM: Computational identification of cis-regulatory elements associated with groups of functionally related genes in Saccharomyces cerevisiae. J Mol Biol 2000, 296(5): I205-I2I4.

27. Hong JK, Lee SC, Hwang BK: Activation of pepper basic PR-I gene promoter during defense signaling to pathogen, abiotic and environmental stresses. Gene 2005, 356:169-180.

28. Lee SC, Kim SH, An SH, Yi SY, Hwang BK: Identification and functional expression of the pepper pathogen-induced gene, CAPIP2, involved in disease resistance and drought and salt stress tolerance. Plant Mol Biol 2006, 62(I-2):I5I-I64.

29. Rairdan GJ, Delaney TP: Role of salicylic acid and NIMI/NPRI in race-specific resistance in arabidopsis. Genetics 2002, |6 |(2):803-8| I.

30. de Ruijter NCA, Verhees J, van Leeuwen W, Krol AR van der: Evaluation and Comparison of the GUS, LUC and GFP Reporter Systems for Gene Expression Studies in Plants. Plant Bio 2003 5:103-115

31. Delaney T, Uknes S, Vernooij B, Friedrich L, Weymann K, Negrotto D, Gaffney T, Gut-Rella M, Kessman H, Ward E, Ryals J: A central role of salicylic acid in plant disease resistance. Science 1994 , 266:1247-1250.

32. Katagiri F: A global view of defense gene expression regulation - a highly interconnected signaling network. Current Opinion in Plant Biology 2004, 7:506-5II.

33. Lebel E, Heifetz P, Thorne L, Uknes S, Ryals J, Ward E: Functiona analysis of regulatory sequences controlling PR-I gene expression in Arabidopsis. Plant J 1998, 16(2):223-233.

34. Rushton PJ, Tovar Torres J, Parniske M, Wernert P, Hahlbrock K, Somssich IE: Interaction of elicitor-induced DNA-binding proteins with elicitor response elements in the promoters of parsley PRI genes. The EMBO Journal 1996, I5(20):5690-5700.

35. Zipfel C, Robatzek S, Navarro L, Oakeley EJ, Jones JD, Felix G, Boller $\mathrm{T}$ : Bacterial disease resistance in Arabidopsis through flagellin perception. Nature 2004, 428(6984):764-767.

36. Wang D, Amornsiripanitch N, Dong X: A Genomic Approach to Identify Regulatory Nodes in the Transcriptional Network of Systemic Acquited Resistance in Plants. PLoS Pathog 2006, 2(II):el23.

37. Keller B, Baumgartner C: Vascular-specific expression of the bean GRP I.8 gene is negatively regulated. Plant Cell I99I, 3(10): $|05|-|06|$.

38. Bruce WB, Deng XW, Quail PH: A negatively acting DNA sequence element mediates phytochrome-directed repression of phyA gene transcription. Embo J |99|, I0(I0):30I5-3024

39. Tepperman JM, Hwang YS, Quail PH: phyA dominates in transduction of red-light signals to rapidly responding genes at the initiation of Arabidopsis seedling de-etiolation. Plant J 2006, 48(5):728-742.

40. Molina C, Grotewold E: Genome wide analysis of Arabidopsis core promoters. BMC Genomics 2005, 6:

4I. Michael TP, Mockler TC, Breton G, McEntee C, Byer A, Trout JD Hazen SP, Shen R, Priest HD, Sullivan CM, Givan SA, Yanovsky M, Hong F, Kay SA, Chory J: Network discovery pipeline elucidates conserved time-of-day-specific cis-regulatory modules. PLOS Genet 2008, 4(2):el4

42. Roberts MR, Paul ND: Seduced by the dark side: integrating molecular and ecological perspectives on the influence of light on plant defence against pests and pathogens. New Phytol 2006, $170(4): 677-699$.

43. Dietrich RA, Delaney TP, Uknes SJ, Ward EJ, Ryals JA, Dangl JL: Arabidopsis mutants simulating disease resistance response. Cell 1994, 77:565-578.

44. Dangl JL, Dietrich RA, Richberg MH: Death Don't Have No Mercy: cell death programs in plant-microbe interactions. Plant Cell 1996, 8: 1793-1807.

45. Torres MA, Dangl JL: Functions of the respiratory burst oxidase in biotic interactions, abiotic stress and development. Curr Opin Plant Biol 2005, 8(4):397-403.

46. Jabs T, Colling C, Tschöpe M, Hahlbrock K, Scheel D: Elicitor-stimulated ion fluxes and reactive oxygen species from the oxidative burst signal defense gene activation and phytoalexin synthesis in parsley. Proc Natl Acad Sci USA 1997, 94:4800-4805.

47. Shirasu K, Nakajima H, Rajasekhar VK, Dixon RA, Lamb CJ: Salicylic acid potentiates an agonist-dependent gain control that amplifies pathogen signals in the activation of defense mechanisms. Plant Cell 1997, 9:261-270.

48. Taylor JE, McAinsh MR: Signalling crosstalk in plants: emerging issues. J Exp Bot 2004, 55(395): I47-I49.

49. McDowell JM, Cuzick A, Can C, Beynon J, Dangl JL, Holub EB: Downy mildew (Peronospora parasitica) resistance genes in Arabidopsis vary in functional requirements for NDRI, EDSI, NPRI, and Salicylic Acid accumulation. Plant / 2000, 22:523-530.

50. Desveaux D, Subramaniam R, Despres C, Mess JN, Levesque C, Fobert PR, Dangl JL, Brisson N: A "Whirly" transcription factor is required for salicylic acid-dependent disease resistance in Arabidopsis. Dev Cell 2004, 6(2):229-240.

51. Sprenger-Haussels M, Weisshaar B: Transactivation properties of parsley proline-rich bZIP transcription factors. Plant J 2000, 22(I): I-8

52. Popescu SC, Popescu GV, Bachan S, Zhang Z, Seay M, Gerstein M, Snyder M, Dinesh-Kumar SP: Differential binding of calmodulinrelated proteins to their targets revealed through high-den- 
sity Arabidopsis protein microarrays. Proc Natl Acad Sci USA 2007, 104(II):4730-4735.

53. Sambrook J, Fritsch S, Maniatis T: Molecular cloning: A laboratory manual. Volume 2. Cold Spring Harbor: Cold Spring HarborPress; 1989.

54. Clough SJ, Bent AF: Floral dip: A simplified method for Agrobacterium-mediated transformation of Arabidopsis thaliana. Plant J 1998, 16:735-743.

55. Hruz T, Laule O, Szabo G, Wessendorp F, Bleuler S, Oertle L, Widmayer P, Gruissem W, Zimmermann P: Genevestigator V3: a reference expression database for the meta-analysis of transcriptomes. Advances in Bioinformatics 2008:420747.

56. Kleine $T$, Kindgren $P$, Benedict $C$, Hendrickson L, Strand $A$ : Genome-wide gene expression analysis reveals a critical role for CRYPTOCHROMEI in the response of Arabidopsis to high irradiance. Plant Physiol 2007, I44(3): |39|- 406.

Publish with Bio Med Central and every scientist can read your work free of charge

"BioMed Central will be the most significant development for disseminating the results of biomedical research in our lifetime. "

Sir Paul Nurse, Cancer Research UK

Your research papers will be:

- available free of charge to the entire biomedical community

- peer reviewed and published immediately upon acceptance

- cited in PubMed and archived on PubMed Central

- yours - you keep the copyright

Submit your manuscript here:

http://www.biomedcentral.com/info/publishing_adv.asp
BioMedcentral 